\title{
Self-amplified spontaneous emission FEL with energy-chirped electron beam and its application for generation of attosecond $x$-ray pulses
}

\author{
E. L. Saldin, E. A. Schneidmiller, and M. V. Yurkov \\ Deutsches Elektronen-Synchrotron (DESY), Hamburg, Germany
}

(Received 17 March 2006; published 3 May 2006)

\begin{abstract}
Influence of a linear energy chirp in the electron beam on a self-amplified spontaneous emission (SASE) Free Electron Laser (FEL) operation is studied analytically and numerically using a 1D model. Analytical results are based on the theoretical background developed by Krinsky and Huang [Phys. Rev. ST Accel. Beams 6, 050702 (2003)]. Explicit expressions for Green's functions and for output power of a SASE FEL are obtained for the high-gain linear regime in the limits of small and large energy chirp parameters. Saturation length and power versus energy chirp parameter are calculated numerically. It is shown that the effect of linear energy chirp on FEL gain is equivalent to the linear undulator tapering (or linear energy variation along the undulator). A consequence of this fact is a possibility to perfectly compensate FEL gain degradation, caused by the energy chirp, by means of the undulator tapering independently of the value of the energy chirp parameter. An application of this effect for generation of attosecond pulses from a hard $\mathrm{x}$-ray FEL is proposed. Strong energy modulation within a short slice of an electron bunch is produced by a few-cycle optical laser pulse in a short undulator, placed in front of the main undulator. Gain degradation within this slice is compensated by an appropriate undulator taper while the rest of the bunch suffers from this taper and does not lase. Three-dimensional simulations predict that short (200 attoseconds) high-power (up to $100 \mathrm{GW}$ ) pulses can be produced in Angstrom wavelength range with a high degree of contrast. A possibility to reduce pulse duration to sub-100 attosecond scale is discussed.
\end{abstract}

DOI: 10.1103/PhysRevSTAB.9.050702

PACS numbers: 41.60.Cr, 41.50.+h, 42.55.Vc

\section{INTRODUCTION}

Start-to-end simulations [1] of the TESLA Test Facility Free Electron Laser (TTF FEL), Phase 1 [2], have shown the presence of a strong energy chirp (energy-time correlation) within a short high-current leading peak in electron density distribution that has driven the self-amplified spontaneous emission (SASE) FEL process. The energy chirp was accumulated due to the longitudinal space charge after compression. According to the simulations (that reproduced well the measured FEL properties), the energy chirp had a dramatic impact on SASE FEL saturation length and output characteristics. A similar effect takes place during the operation of VUV FEL at DESY in a "femtosecond mode" [3-5]. Such a mode of operation might also be possible in future $x$-ray SASE FELs.

There also exists a concept of frequency-chirped SASE FELs (frequency chirp of SASE FEL radiation is correlated with energy chirp in the electron beam due to the FEL resonance condition) aiming at the shortening of radiation pulse with the help of a monochromator [6]. Energy chirp can also be used to tune the output frequency of an FEL with coherent prebunching as demonstrated in the experiment at the DUV FEL facility [7]. Thus, a theoretical understanding of the energy chirp effect on the FEL performance is of crucial importance.

Analytical studies on this subject were performed in [8] in the framework of one-dimensional approximation. The general form of a time-domain Green's function as an inverse Laplace transform was derived in [8]. It was then reduced to the explicit expression in the limit of small energy chirp parameter up to the first order, resulting in phase correction (and ignoring the gain correction). This explicit solution for the Green's function was used to analyze statistical properties of a chirped SASE FEL in this limit. A second-order correction to the FEL gain was presented in [6] but this result is incorrect.

In this paper we study the impact of energy chirp on SASE FEL performance. We also find that FEL gain degradation can be perfectly compensated by undulator tapering. We discuss an application of the compensation effect for generation of attosecond pulses from x-ray FELs such as European XFEL [9] and Linac Coherent Light Source [10].

\section{GREEN'S FUNCTION}

Let us consider a planar undulator with the magnetic field

$$
H_{z}(z)=H_{\mathrm{w}} \cos \left(2 \pi z / \lambda_{\mathrm{w}}\right),
$$

where $\lambda_{\mathrm{w}}$ is undulator period, and $H_{\mathrm{w}}$ is peak magnetic field. Electric field of the amplified electromagnetic wave is presented in the form

$$
E=\tilde{E} \exp \left[i \omega_{0}(z / c-t)\right]+\text { c.c., }
$$

where $\omega_{0}$ is a reference frequency and $\tilde{E}$ is slowly varying amplitude [11]. As it was shown in [8], for a SASE FEL, driven by an electron beam with linear energy chirp, $\tilde{E}$ can be written as follows (we use notations from [11]): 


$$
\tilde{E}=2 E_{0} \sum_{j} e^{-i \hat{s}_{j} / \rho} e^{2 i \hat{\alpha} \hat{s}_{j}\left(\hat{s}-\hat{z} / 2-\hat{s}_{j}\right)} g\left(\hat{z}, \hat{s}-\hat{s}_{j}, \hat{\alpha}\right) .
$$

Here $\rho=\lambda_{\mathrm{w}} \Gamma /(4 \pi)$ is the efficiency parameter, $\Gamma^{3}=$ $\pi j_{0} K^{2} A_{J J}^{2} /\left(I_{A} \lambda_{\mathrm{w}} \gamma_{0}^{3}\right), j_{0}$ is the beam current density, $I_{A}=$ $m c^{3} / e \simeq 17 \mathrm{kA}, \quad \gamma_{0}$ is relativistic factor, $K=$ $e \lambda_{\mathrm{w}} H_{\mathrm{w}} /\left(2 \sqrt{2} \pi m c^{2}\right)$ is the rms undulator parameter, $A_{J J}=$ $J_{0}(Q)-J_{1}(Q)$ is the Bessel function factor, $Q=$ $K^{2} /\left[2\left(1+K^{2}\right)\right], E_{0}=\rho \Gamma \gamma_{0}^{2} m c^{2} /\left(e K A_{J J} \sqrt{2}\right)$ is the saturation field amplitude, $\hat{z}=\Gamma z$ is a normalized position along the undulator, $\hat{s}=\rho \omega_{0}\left(z / \bar{v}_{z 0}-t\right)$ is normalized position along the electron bunch, and $\bar{v}_{z 0}$ is the average longitudinal velocity (defined for a reference particle). Let the energy linearly depend on a particle position in the bunch (or arrival time). The energy chirp parameter

$$
\hat{\alpha}=-\frac{d \gamma}{d t} \frac{1}{\gamma_{0} \omega_{0} \rho^{2}}
$$

is defined such that, for positive sign of $\hat{\alpha}$, particles in the head of the bunch have larger energy than those in the tail. Relativistic factor $\gamma_{0}$ for a reference particle (placed at $\hat{s}=$ 0 ) and reference frequency $\omega_{0}$ are connected by the FEL resonance condition: $\omega_{0}=2 c k_{w} \gamma_{0}^{2} /\left(1+K^{2}\right)$. Note that the theory is applicable when $\rho \hat{\alpha} \ll 1$ [8]. It is also useful to define normalized detuning [11]: $\hat{C}=\left[k_{w}-\omega(1+\right.$ $\left.\left.K^{2}\right) / 2 c \gamma_{0}^{2}\right] / \Gamma$.

The Green's function $g$, entering Eq. (1), is given by the inverse Laplace transform [8]:

$$
g(\hat{z}, \hat{s}, \hat{\alpha})=2 \int_{\gamma^{\prime}-i \infty}^{\gamma^{\prime}+i \infty} \frac{d p}{2 \pi i p} \exp [f(p, \hat{z}, \hat{s}, \hat{\alpha})],
$$

where

$$
f(p, \hat{z}, \hat{s}, \hat{\alpha})=p(\hat{z}-2 \hat{s})+\frac{2 i \hat{s}}{p(p+i \hat{\alpha} \hat{s})} .
$$

We use a saddle point approximation to get an estimate of the integral (3) for large values of $\hat{z}$ [8]. The saddle point is determined from the condition $f^{\prime}=0$ which leads to the 4th power equation with three parameters:

$$
p^{4}+2 i \hat{\alpha} \hat{s} p^{3}-\hat{\alpha}^{2} \hat{s}^{2} p^{2}-\frac{4 i \hat{s}}{\hat{z}-2 \hat{s}} p+\frac{2 \hat{\alpha} \hat{s}^{2}}{\hat{z}-2 \hat{s}}=0 .
$$

Once the saddle point, $p_{0}$, is found, the Green's function can be approximated as follows:

$$
g(\hat{z}, \hat{s}, \hat{\alpha})=\frac{2 \exp \left[f\left(p_{0}, \hat{z}, \hat{s}, \hat{\alpha}\right)\right]}{p_{0}\left[2 \pi f^{\prime \prime}\left(p_{0}, \hat{z}, \hat{s}, \hat{\alpha}\right)\right]^{1 / 2}} .
$$

Let us first consider the case when the energy chirp is a small perturbation, $|\hat{\alpha}| \hat{z} \ll 1, \hat{z} \gg 1$. A second-order expansion of the Green's function takes the following form:

$$
\begin{aligned}
g(\hat{z}, \hat{s}, \hat{\alpha}) \simeq & \frac{e^{-i \pi / 12}}{\sqrt{\pi \hat{z}}} \exp \left[i^{1 / 3} \hat{z}+i^{2 / 3} \frac{\hat{\alpha} \hat{s}}{2}\left(1+i \frac{\hat{\alpha} \hat{z}^{2}}{36}\right)\right. \\
& -9 i^{1 / 3}\left(1-\frac{\hat{\alpha}^{2} \hat{z}^{2}}{216 i^{2 / 3}}\right) \frac{(\hat{s}-\hat{z} / 6)^{2}}{\hat{z}} \\
& \left.-\frac{i}{2} \hat{\alpha} \hat{s}(\hat{z}-2 \hat{s})\right] .
\end{aligned}
$$

The leading correction term is the last term in the argument of the exponential function. This term was found in [8] (note the difference in the definition of normalized parameters). Setting $\hat{\alpha}=0$, one gets from (7) the well-known Green's function for unchirped beam [12].

Now let us consider the case $\hat{\alpha}>0$ and $1 \ll \hat{\alpha} \ll \hat{z}$. The Green's function for $\hat{s} \gg \hat{\alpha}^{-1}$ is approximated by

$$
g(\hat{z}, \hat{s}, \hat{\alpha}) \simeq\left(\frac{\hat{\alpha}}{2 \pi^{2} \hat{z}}\right)^{1 / 4} \exp \left(2 \sqrt{\frac{2 \hat{z}}{\hat{\alpha}}}-2 \sqrt{\frac{2}{\hat{\alpha} \hat{z}}} \hat{s}\right) .
$$

More thorough analysis for small values of $\hat{s}$ shows that the Green's function has a maximum at $\hat{s}_{\mathrm{m}}=2^{1 / 3} \hat{\alpha}^{-1}$, i.e., the position of maximum is independent of $\hat{z}$ while the width of the radiation wave packet is proportional to $\sqrt{\hat{\alpha}} \hat{z}$. The mean frequency of the radiation wave packet corresponds to a resonant frequency at $\hat{s}=0$. Note also that the beam density excitation is concentrated near $\hat{s}=0$ within a much shorter range, of the order of $\hat{\alpha}^{-7 / 4} \hat{z}^{-1 / 4}$.

In the case of $\hat{\alpha}<0$ and $1 \ll|\hat{\alpha}| \ll \hat{z}$, the Green's function is given by

$$
\begin{aligned}
g(\hat{z}, \hat{s}, \hat{\alpha}) \simeq & \frac{2^{1 / 4} e^{-i \pi / 2}}{\pi^{1 / 2}|\hat{\alpha}|^{5 / 4} \hat{z}^{3 / 4} \hat{s}} \exp \left(2 \sqrt{\frac{2 \hat{z}}{|\hat{\alpha}|}}+i|\hat{\alpha}| \hat{z} \hat{s}+\frac{2 i}{|\hat{\alpha}|^{2} \hat{s}}\right. \\
& \left.-\frac{2 \sqrt{2}}{|\hat{\alpha}|^{7 / 2} \hat{z}^{1 / 2} \hat{s}^{2}}-2 \sqrt{\frac{2}{|\hat{\alpha}| \hat{z}} \hat{s}}\right) .
\end{aligned}
$$

The width of the radiation wave packet (and of the beam density excitation as well) is of the order of $|\hat{\alpha}|^{-7 / 4} \hat{z}^{-1 / 4}$. The maximum of the wave packet is positioned at $\hat{s}_{\mathrm{m}}=$ $2^{5 / 4}|\hat{\alpha}|^{-7 / 4} \hat{z}^{-1 / 4}$, i.e., the wave packet is shrinking and back-propagating (with respect to the electron beam) with increasing $\hat{z}$. The mean frequency of the wave packet is blueshifted with respect to resonant frequency at $\hat{s}=0$. In normalized form, this shift is $\Delta \hat{C}=-|\hat{\alpha}| \hat{z} / 2$.

\section{LINEAR REGIME OF SASE FEL}

The normalized radiation power (normalized efficiency), $\langle\hat{\eta}\rangle=P_{\mathrm{SASE}} / \rho P_{\text {beam }}$, can be expressed as follows [11]:

$$
\langle\hat{\eta}\rangle=\frac{\left\langle|\tilde{E}|^{2}\right\rangle}{4 E_{0}^{2}},
$$

where $\langle\cdots\rangle$ means ensemble average. One can easily get from (1) 


$$
\langle\hat{\eta}(\hat{z}, \hat{\alpha})\rangle=\frac{1}{N_{\mathrm{c}}} \int_{0}^{\infty} d \hat{s}|g(\hat{z}, \hat{s}, \hat{\alpha})|^{2} .
$$

Here $N_{\mathrm{c}}=N_{\lambda} /(2 \pi \rho)$ is a number of cooperating electrons (populating $\Delta \hat{s}=1$ ), $N_{\lambda}$ is a number of electrons per wavelength. The local power growth rate [13] can be computed as follows:

$$
G(\hat{z}, \hat{\alpha})=\frac{d}{d \hat{z}} \ln \langle\hat{\eta}(\hat{z}, \hat{\alpha})\rangle .
$$

Applying Eqs. (11) and (12) to the asymptotical cases, considered in the previous section, we get the following results. For the case $|\hat{\alpha}| \hat{z} \ll 1, \hat{z} \gg 1$ the FEL power is given by

$$
\langle\hat{\eta}\rangle \simeq \frac{\exp \left\{\sqrt{3} \hat{z}\left[1-(\hat{\alpha} \hat{z} / 12)^{2} / 3\right]+\hat{\alpha} \hat{z} / 12\right\}}{3^{5 / 4} \sqrt{\pi \hat{z}} N_{\mathrm{c}}}
$$

and the local power growth rate is

$$
G(\hat{z}, \hat{\alpha}) \simeq \sqrt{3}\left[1-\left(\frac{\hat{\alpha} \hat{z}}{12}\right)^{2}\right]-\frac{1}{2 \hat{z}}+\frac{\hat{\alpha}}{12} .
$$

It reaches maximum $G_{\mathrm{m}}=\sqrt{3}\left[1-(|\hat{\alpha}| / 16)^{2 / 3}\right]+\hat{\alpha} / 12$ at the position $\hat{z}_{\mathrm{m}}=3^{1 / 2} 2^{2 / 3} /|\hat{\alpha}|^{2 / 3}$. Numerical simulations show that Eqs. (13) and (14) are pretty accurate up to the values $|\hat{\alpha}| \hat{z}$ of the order of unity although the condition $|\hat{\alpha}| \hat{z} \ll 1$ was used to derive them.

For the case $\hat{\alpha}>0$ and $1 \ll \hat{\alpha} \ll \hat{z}$, we get rather simple expressions:

$$
\begin{gathered}
\langle\hat{\eta}(\hat{z}, \hat{\alpha})\rangle \simeq \frac{\hat{\alpha}}{8 \pi N_{\mathrm{c}}} \exp \left(4 \sqrt{\frac{2 \hat{z}}{\hat{\alpha}}}\right), \\
G(\hat{z}, \hat{\alpha}) \simeq 2 \sqrt{\frac{2}{\hat{\alpha} \hat{z}}}
\end{gathered}
$$

For large negative values of $\hat{\alpha}$ we obtain

$$
\begin{gathered}
\langle\hat{\eta}\rangle \simeq \frac{1}{2^{7 / 4} \pi^{1 / 2}|\hat{\alpha}|^{3 / 4} \hat{z}^{5 / 4} N_{\mathrm{c}}} \exp \left(4 \sqrt{\frac{2 \hat{z}}{|\hat{\alpha}|}}\right), \\
G(\hat{z}, \hat{\alpha}) \simeq 2 \sqrt{\frac{2}{|\hat{\alpha}| \hat{z}}}-\frac{5}{4 \hat{z}} .
\end{gathered}
$$

\section{NONLINEAR REGIME}

We studied the nonlinear regime of a chirped SASE FEL operation with 1D version of the code FAST $[11,14]$. Analytical results, presented above, were used as a primary standard for testing the code in linear regime. Green's function was modeled by exciting density modulation on a short scale, $\Delta \hat{s} \ll 1$. SASE FEL initial conditions were simulated in a standard way [11]. The results of numerical simulations in all cases were in good agreement with analytical results presented in two previous sections.

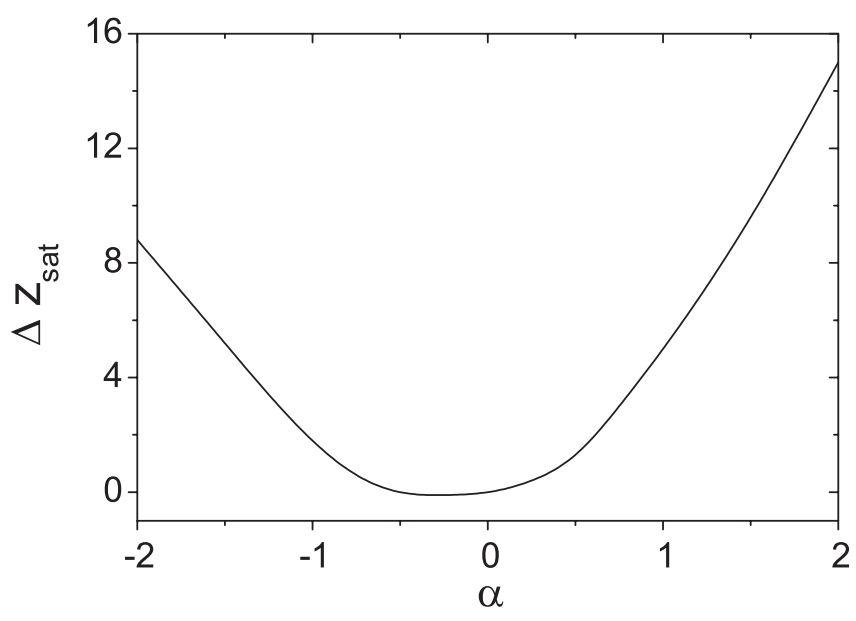

FIG. 1. Increase of saturation length $\Delta \hat{z}_{\text {sat }}=\hat{z}_{\text {sat }}(\hat{\alpha})-\hat{z}_{\text {sat }}(0)$ versus parameter $\hat{\alpha}$. Here $\hat{z}_{\text {sat }}(0)=13$.

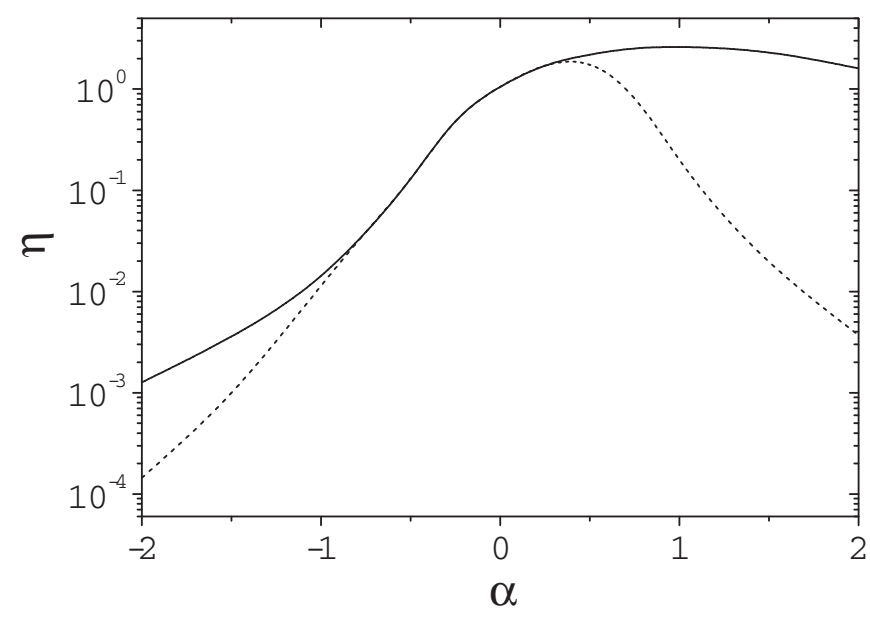

FIG. 2. Normalized output power versus parameter $\hat{\alpha}$. Solid: $\hat{z}=\hat{z}_{\text {sat }}(\hat{\alpha})$ (see Fig. 1); dashed: $\hat{z}=\hat{z}_{\text {sat }}(0)=13$.

The main results of the simulations of the nonlinear regime are presented in Figs. 1 and 2. Saturation length and power are functions of two parameters, $\hat{\alpha}$ and $N_{\mathrm{c}}$. For our simulations we have chosen $N_{\mathrm{c}}=3 \times 10^{7}-$ a typical value for VUV SASE FELs. Note, however, that the results, presented in Figs. 1 and 2, very weakly depend on $N_{\mathrm{c}}$. Figure 1 shows increase of saturation length with respect to unchirped beam case. In Fig. 2 the output power is plotted versus chirp parameter for two cases: when undulator length is equal to a saturation length for a given $\hat{\alpha}$ and when it is equal to the saturation length for the unchirped beam case. One can see sharp reduction of power for negative $\hat{\alpha}$ while a mild positive chirp $(\hat{\alpha}<0.5)$ is beneficial for SASE.

\section{ENERGY CHIRP AND UNDULATOR TAPERING}

Let us consider now the case when there is no energy chirp $(\hat{\alpha}=0)$ and the detuning parameter changes linearly 
along the undulator [11]: $\hat{C}(\hat{z})=\hat{b}_{1} \hat{z}$. This change can be due to variation of undulator parameters $[K(\hat{z})$ and/or $\left.k_{\mathrm{w}}(\hat{z})\right]$, or due to an energy change $\gamma_{0}(\hat{z})$. We have found from numerical simulations that in such a case the effect on FEL gain is exactly the same as in the case of energy chirp and no taper if $\hat{\alpha}=2 \hat{b}_{1}$ for any value of $\hat{\alpha}$ (Fig. 3 shows an example). Therefore, all the results of two previous sections can be also used for the case of linear variation of energy or undulator parameters with the substitution $\hat{\alpha} \rightarrow$ $2 \hat{b}_{1}$. The amplitudes of Green's functions are also the same while the phases are obviously different. In case of $\hat{b}_{1}=0$, $\hat{\alpha} \neq 0$ there is a frequency chirp along the bunch while in the case $\hat{b}_{1}=0, \hat{\alpha} \neq 0$ the frequency is changing along the undulator.

An effect of undulator tapering (or energy change along the undulator) on FEL gain was studied in [13] in the limit $\hat{b}_{1} \ll 1$. Comparing our Eq. (13) (with the substitution $\hat{\alpha} \rightarrow 2 \hat{b}_{1}$ ) and Eq. (45) of Ref. [13], we can see that quadratic correction term in the argument of the exponential function is the same but the linear term is 2 times larger in [13]. The reason for discrepancy is that the frequency dependence of the preexponential factor in Eq. (42) of Ref. [13] is neglected.

A symmetry between two considered effects (energy chirp and undulator tapering) can be understood as follows. If we look at the radiation field acting on some test electron from an electron behind it, this field was emitted at a retarded time. In the first case, a back electron has a detuning due to an energy offset, in the second case it has the same detuning because undulator parameters were different at a retarded time. The question arises: can these two effects compensate each other? We give a positive answer based on numerical simulations (see Fig. 3 as an example): by setting $\hat{b}_{1}=-\hat{\alpha} / 2$ we get rid of gain

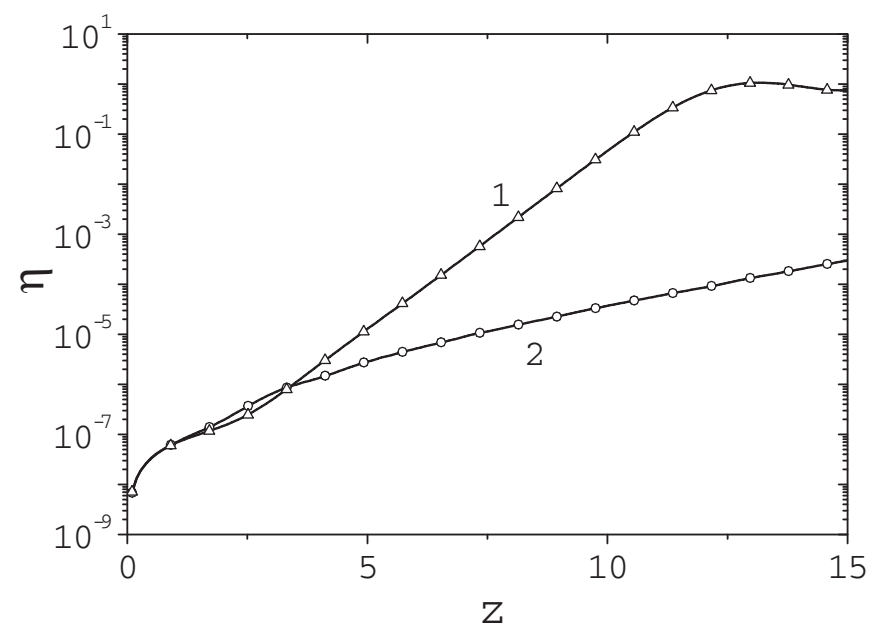

FIG. 3. Normalized power versus undulator length. Solid line 1: $\hat{\alpha}=0, \hat{b}_{1}=0$; triangles: $\hat{\alpha}=4, \hat{b}_{1}=-2$; solid line $2: \hat{\alpha}=$ $4, \hat{b}_{1}=0$; circles: $\hat{\alpha}=0, \hat{b}_{1}=2$. degradation, and FEL power at any point along the undulator is the same as in the case of unchirped beam and untapered undulator. This holds for any value of $\hat{\alpha}$. For instance, if one linearly changes magnetic field $H_{w}$ of the undulator, the compensation condition can be written as follows (nominal values of parameters are marked with subscript “0”):

$$
\frac{1}{H_{\mathrm{w} 0}} \frac{d H_{\mathrm{w}}}{d z}=-\frac{1}{2} \frac{\left(1+K_{0}^{2}\right)^{2}}{K_{0}^{2}} \frac{1}{\gamma_{0}^{3}} \frac{d \gamma}{c d t} .
$$

Of course, in such a case we get frequency-chirped SASE pulse. Since compensation of gain degradation is possible also for large values of $\hat{\alpha}$ (there is no theoretical limit on the value of chirp parameter, except for above-mentioned condition $\rho \hat{\alpha} \ll 1$ ), one can, in principle, organize a regime when a frequency chirp within an intensity spike is much larger than the natural FEL bandwidth (given by $\left.\rho \omega_{0}\right)$.

\section{GENERATION OF ATTOSECOND PULSES}

Up to now several schemes for generation of attosecond pulses from x-ray SASE FELs have been proposed [15$20]$. Here we mention the schemes considered in $[17,18]$ making use of energy modulation of a short slice in the electron bunch by a high-power few-cycle optical pulse in a two-period undulator. Because of energy modulation the frequency of SASE radiation in the x-ray undulator is correlated to the longitudinal position within the fewcycle-driven slice of the electron beam. The largest frequency offset corresponds to a single-spike pulse in time domain (about 300 attoseconds). The selection of singlespike pulses is achieved by using a crystal monochromator after the x-ray undulator [17], or with the help of the other undulator tuned to the offset frequency [18].

In this paper we propose a new scheme (see Fig. 4) that makes use of the compensation effect, described in the previous section. Indeed, there is a strong energy chirp around zero-crossing of energy modulation. If one uses appropriate undulator taper then only a short slice around

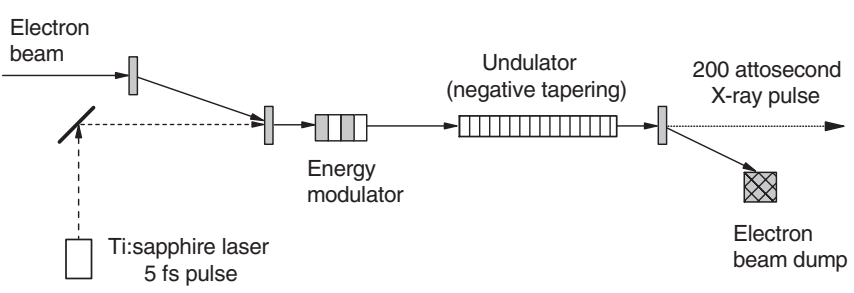

FIG. 4. Schematic diagram of the attosecond x-ray source. The energy modulator performs slice energy modulation of the electron bunch (see Fig. 6). The undulator tapering leads to complete suppression of the amplification process in the largest fraction of the electron bunch, and the output x-ray pulse has 200 attosecond pulse duration. 
zero-crossing produces powerful FEL pulse. The main part of the bunch is unmodulated and suffers from strong negative undulator tapering (see Fig. 2). One should also note that for large negative taper the SASE FEL gain is very sensitive to longitudinal velocity spread. Therefore, a highcontrast attosecond pulse is directly produced in the undulator.

Operation of attosecond SASE FEL is illustrated for the parameters close to those of the European XFEL operating at the wavelength $0.15 \mathrm{~nm}$ [9]. The parameters of the electron beam are: energy $15 \mathrm{GeV}$, charge $1 \mathrm{nC}$, rms pulse length $25 \mu \mathrm{m}$, rms normalized emittance $1.4 \mathrm{~mm}-\mathrm{mrad}$, rms energy spread $1 \mathrm{MeV}$. Undulator period is $3.65 \mathrm{~cm}$.

The parameters of the seed laser are: wavelength $800 \mathrm{~nm}$, energy in the laser pulse $3 \mathrm{~mJ}$, and FWHM pulse duration 5 fs (see Fig. 5). The laser beam is focused onto the electron beam in a short undulator resonant at the optical wavelength of $800 \mathrm{~nm}$. Optimal conditions of the focusing correspond to the positioning of the laser beam waist in the center of the modulator undulator. It is assumed that the phase of laser field corresponds to "sine" mode (dashed line with $\varphi=\pi / 2$, see Fig. 5). Parameters of the modulator undulator are: period length $50 \mathrm{~cm}$, peak field $1.6 \mathrm{~T}$, number of periods 2 . The interaction with the laser light in the undulator produces a time-dependent electron energy modulation as it is shown in Fig. 6. This modulation corresponds to the energy chirp parameter $\hat{\alpha} \simeq$ 2 at zero-crossing ( $t=5 \mathrm{fs}$ fs in Fig. 6).

Optimization of the attosecond SASE FEL has been performed with the three-dimensional, time-dependent code FAST [14] taking into account all physical effects influencing the SASE FEL operation (diffraction effects, energy spread, emittance, slippage effect, etc.). Threedimensional simulations confirmed the predictions of the one-dimensional model: the energy chirp and the undulator tapering compensate each other, there is strong suppression



FIG. 5. Possible evolutions of the electric field in the 5-fs pulse carried at a wavelength $800 \mathrm{~nm}$ for two different pulse phases $(\phi=0, \pi / 2)$.

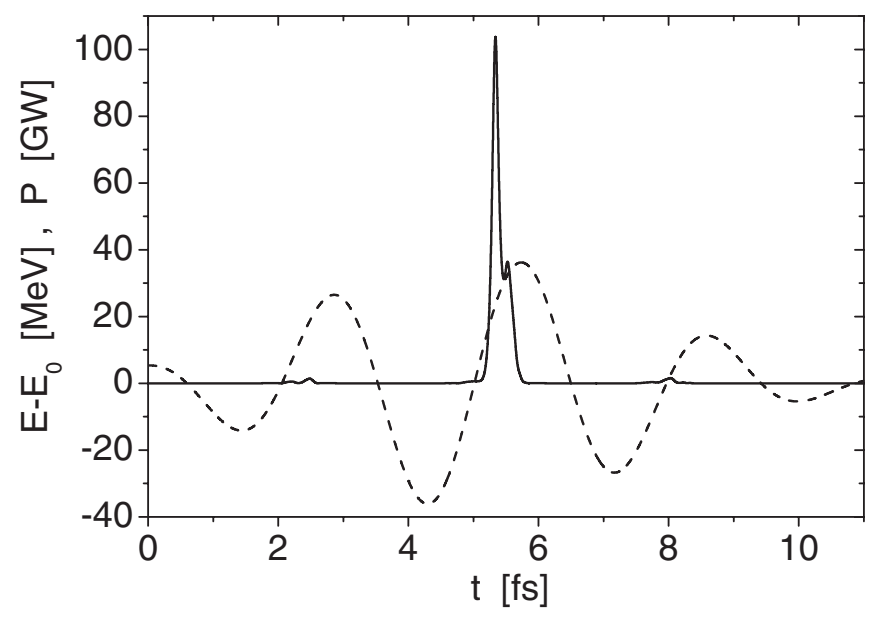

FIG. 6. Energy modulation of the electron beam at the exit of the modulator undulator (dotted line) and a profile of the radiation pulse at the undulator length $100 \mathrm{~m}$.

of the amplification in the case of uncompensated negative taper.

Undulator tapering is performed by changing the gap of undulator modules [9] such that magnetic field increases linearly along the undulator length $\left(\hat{b}_{1}<0\right)$. We performed the scan of tapering depth $\hat{b}_{1}$ in order to maximize the power in the main peak on one hand, and to minimize contribution of the background, on the other hand. We ended up with the value of taper which is about $20 \%$ smaller than that required for a perfect compensation of chirp at $t=5 \mathrm{fs}$. Note that the chirp is not linear in the region of interest. In addition, a mild net positive chirp is beneficial for SASE, as it was discussed above (see Fig. 2).

A typical radiation pulse at the undulator length $100 \mathrm{~m}$ is shown in Fig. 6. One can see a high-power spike in the region where the energy chirp is well compensated by the taper and two weak side peaks at $t \simeq 2 \mathrm{fs}$ and $t \simeq 8 \mathrm{fs}$ where the net effect is negative taper. In the rest of the bunch a large negative taper together with velocity spread and $3 \mathrm{D}$ effects completely suppresses amplification. In Fig. 7 we present three different shots illustrating the properties of the main peak. Typical pulse duration is about 200 attoseconds (FWHM) and peak power ranges from several tens up to hundred GW. To estimate the contrast (which we define as the ratio of energy in the main peak to the total radiated energy at the experiment), we assume that an angular collimation is used in order to reduce spontaneous emission background. A collimator with half-angle $3 \mu \mathrm{rad}$ allows the entire intensity in the main peak to be transmitted. The contrast is influenced by SASE intensity in two side peaks and by spontaneous emission in the first harmonic from the rest of the bunch. For the charge of $1 \mathrm{nC}$, as in our numerical example, the contrast is about 95\%. Higher harmonics of undulator radiation (if they disturb an experiment) can be cut, for instance, by a multilayer monochromator with a bandwidth of the order of $1 \%$. 


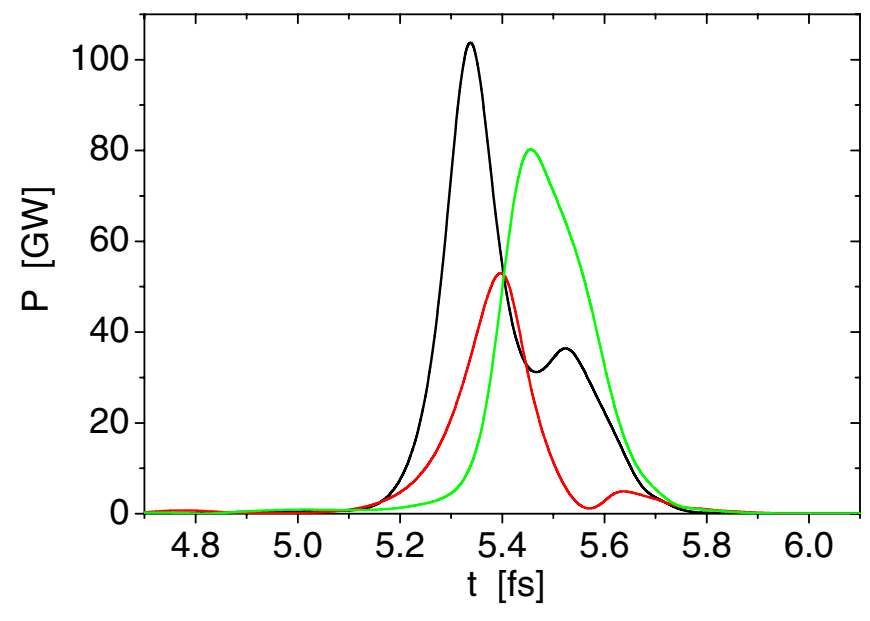

FIG. 7. (Color) Temporal structure of the radiation pulse (three different shots) at the undulator length $100 \mathrm{~m}$.

\section{BEYOND "FUNDAMENTAL LIMIT"}

It is generally accepted that the shortest pulse, that can be obtained from a SASE FEL, is given by a duration of intensity spike in time domain, i.e., it is defined by inverse FEL bandwidth $\left(\rho \omega_{0}\right)^{-1}$. However, the fact that a SASE FEL can operate with a strong chirp parameter (in combination with undulator tapering) without gain degradation opens up a possibility of a conceptual breakthrough: one can get from SASE FEL a radiation pulse which is much shorter than the inverse FEL bandwidth. Indeed, in the case of $\hat{\alpha} \gg 1$, the frequency chirp inside an intensity spike is much larger than FEL bandwidth. Thus, one can use a monochromator to reduce pulse duration. By an appropriate choice of the monochromator bandwidth, one can select an $\mathrm{x}$-ray pulse that is shorter by a factor of $\sqrt{2 \hat{\alpha}}$ than the inverse FEL bandwidth. The only theoretical limit in this case is given by the condition $\rho \hat{\alpha} \ll 1$. Note that for hard x-ray FELs the parameter $\rho$ is in the range $10^{-4}-10^{-3}$.

To illustrate a possible technical realization of this idea, we can suppose that the energy modulation by a few-cycle optical pulse is increased by a factor 3 so that $\hat{\alpha} \simeq 6$. In combination with undulator tapering and a monochromator, this would allow one to obtain sub-100-GW coherent $\mathrm{x}$-ray pulses with a duration below 100 attoseconds and a contrast about $80 \%-90 \%$.

[1] M. Dohlus, K. Floettmann, O. Kozlov, T. Limberg, P. Piot, E. Saldin, E. Schneidmiller, and M. Yurkov, Nucl. Instrum. Methods Phys. Res., Sect. A 530, 217 (2004).

[2] V. Ayvazyan et al., Phys. Rev. Lett. 88, 104802 (2002).

[3] E. Saldin, E. Schneidmiller, and M. Yurkov, DESY Print TESLA-FEL 2004-06, July 2004.

[4] E. Saldin, E. Schneidmiller, and M. Yurkov, DESY Print DESY 05-239, November 2005.

[5] V. Ayvazyan et al., Eur. Phys. J. D 37, 297 (2006).

[6] C. Schroeder, C. Pellegrini, S. Reiche, J. Arthur, and P. Emma, Nucl. Instrum. Methods Phys. Res., Sect. A 483, 89 (2002).

[7] T. Shaftan et al., Proceedings of the 2004 FEL Conference, p. 282.

[8] S. Krinsky and Z. Huang, Phys. Rev. ST Accel. Beams 6, 050702 (2003).

[9] TESLA XFEL: First Stage of the X-ray Laser LaboratoryTechnical Design Report, DESY 2002-167.

[10] SLAC Linac Coherent Light Source (LCLS) Conceptual Design Report, SLAC-R-593, 2002.

[11] E. Saldin, E. Schneidmiller, and M. Yurkov, The Physics of Free Electron Lasers (Springer- Verlag, Berlin, 1999).

[12] S. Krinsky and R. Gluckstern, Phys. Rev. ST Accel. Beams 6, 050701 (2003).

[13] Z. Huang and G. Stupakov, Phys. Rev. ST Accel. Beams 8, 040702 (2005).

[14] E. Saldin, E. Schneidmiller, and M. Yurkov, Nucl. Instrum. Methods Phys. Res., Sect. A 429, 233 (1999).

[15] E. Saldin, E. Schneidmiller, and M. Yurkov, Opt. Commun. 212, 377 (2002).

[16] A. A. Zholents and W. M. Fawley, Phys. Rev. Lett. 92, 224801 (2004).

[17] E. Saldin, E. Schneidmiller, and M. Yurkov, Opt. Commun. 237, 153 (2004).

[18] E. Saldin, E. Schneidmiller, and M. Yurkov, Opt. Commun. 239, 161 (2004).

[19] P. Emma, Z. Huang, and M. Borland, Proceedings of the 2004 FEL Conference, p. 333.

[20] A. Zholents and G. Penn, Phys. Rev. ST Accel. Beams 8, 050704 (2005). 